\title{
16S rRNA monitoring point-of-care magnetic focus lateral flow sensor
}

\author{
Wen Ren, ${ }^{a, b}$ Saeed Ahmad ${ }^{a, b}$ and Joseph Irudayaraj ${ }^{a, b, c, d, *}$
}

a. Department of Bioengineering. University of Illinois at Urbana-Champaign, Urbana, IL, 61801, USA

b. Biomedical Research Center in Mills Breast Cancer Institute, Carle Foundation Hospital, Urbana, IL, 61801, USA

c. Micro and Nanotechnology Laboratory. University of Illinois at Urbana-Champaign, Urbana,

IL, 61801, USA

d. Cancer Center at Illinois (CCIL), University of Illinois at Urbana-Champaign, Urbana, IL, 61801, USA 


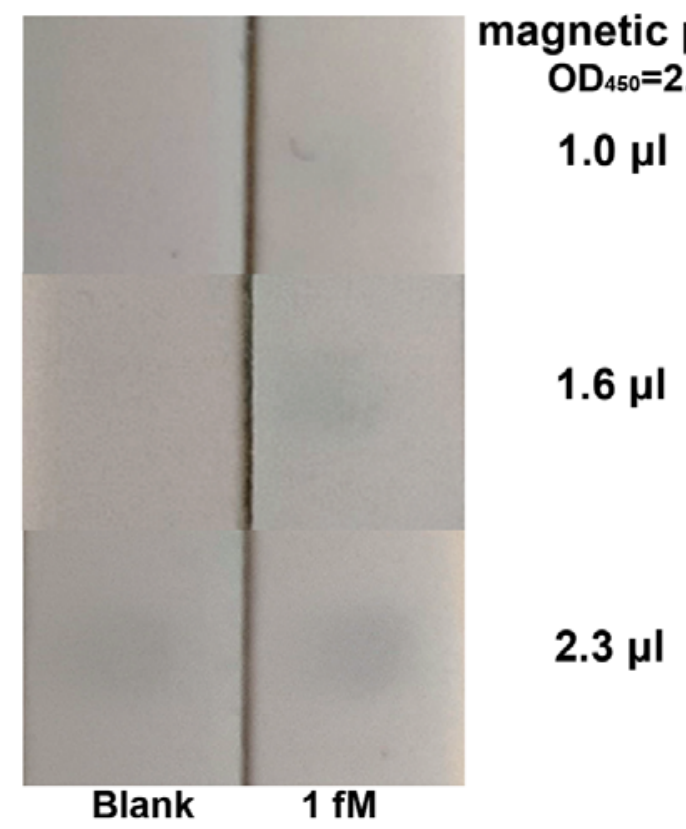

Figure S1. Images of the test zone of the strips from the detection of blank and TS samples with various concentration of magnetic probes. Benefiting from the HRP catalyzed colorimetric reaction, the signal from the proposed mLFS was greatly improved. Even trace amount of nonspecific binding of magnetic probes would induce a color in the test zone of the LF strip due to amplification with HRP conjugated on to the probes. Meanwhile, for the biotinylation and the conjugation of SA-HRP, instead of short oligonucleotide the mNPs were blocked with casein during probe preparation. It is known that strong interaction between gold surface and ssDNA has been utilized to develop label-free detection methods. ${ }^{1-2}$ Therefore, there is a possible non-specific interaction between the magnetic probes and the FITC labeled CS oligonucleotide. In the proposed mLFS method, the antibody conjugated on the LF strip captured the FITC labels. The possible interaction between the FITC-CS and magnetic probes would non-selectively link the probes in the test zone. Thus, the concentration of magnetic probes used for detection was optimized for the best detection performance and minimal nonspecific signal as illustrated in the results from different amount of magnet probes (Figure S1). 
It can be seen that with $1.0 \mu \mathrm{l}$ of magnetic probes with an $\mathrm{OD}_{450}$ of 2.8 measured with nanodrop, the signal from $1 \mathrm{fM}$ of TS was quite weak while there is no observable color from the blank sample. When the concentration of magnetic probes increased to $1.6 \mu 1$, the signal from $1 \mathrm{fM}$ of TS became clearer while the blank sample generated no color in the test zone. There was a notable spot as shown in Figure S1 in the test zone when blank samples were tested with 2.3 $\mu \mathrm{l}$ of magnetic probes, although the signal from $1 \mathrm{fM}$ of TS is clearer.

\section{Reference.}

1. Li, H.; Rothberg, L. J., Label-free colorimetric detection of specific sequences in genomic DNA amplified by the polymerase chain reaction. J. Am. Chem. Soc. 2004, 126 (35), 1095810961.

2. Lv, Z.; Wei, H.; Li, B.; Wang, E., Colorimetric recognition of the coralyne-poly (dA) interaction using unmodified gold nanoparticle probes, and further detection of coralyne based upon this recognition system. Analyst 2009, 134 (8), 1647-1651. 